\title{
Análise do conhecimento dos doentes de asma sobre a patologia antes e depois de uma intervenção educativa
}

\section{DOI: 10.47224/rm.v5i10.89}

\author{
Sara Tamna Venutura Melo \\ Brenda Tolentino Costa do Carmo \\ Giulia Messias Prado \\ Isabella Guerra Araújo \\ Isadora Viana Veiga \\ Kátia Gomes Peixoto \\ Larissa Gomes Espinosa \\ Natália Rabelo Gonzaga \\ Herbert Cristian de Souza \\ Vanessa Silva Lemos
}

e-mail: sara_tamna@hotmail.com

\section{Resumo}

A asma é uma doença inflamatória crônica associada à hiperresponsividade das vias aéreas e obstrução ao fluxo aéreo, seu quadro clínico é caracterizado por episódios recorrentes de sibilos, dispneia, opressão torácica e tosse. O objetivo deste trabalho foi analisar o conhecimento de pacientes portadores de asma a respeito da patologia, antes e depois de uma intervenção educativa. Trata-se de um estudo descritivo de corte transversal com abordagem qualitativa por meio de aplicação de questionários. Os critérios de inclusão foram pacientes de 18 a 60 anos atendidos por um Centro Ambulatorial com o diagnóstico de asma registrado em prontuário e que concordaram em participar do estudo em todas as suas fases. Foram excluídos os que não consentirem em responder ao questionário, e/ou que apresentaram déficit cognitivo e com outros diagnósticos pulmonares que não asma. O estudo demonstrou que os pacientes que participaram das reuniões obtiveram resultados positivos, como a melhoria do conhecimento e da gestão da doença. A realização das reuniões com um pequeno número de participantes contribuiu para maior adaptação dos temas abordados às necessidades de cada caso, embora tenha restringido o debate e a troca de conhecimento entre os pacientes.

Palavras-chave: Asma; Autocuidado; Educação em saúde.

\begin{abstract}
Asthma is a chronic inflammatory disease associated with airway hyperresponsiveness and airflow obstruction, its clinical picture is characterized by recurrent episodes of wheezing, dyspnoea, chest tightness and cough. The aim of this work was to analyze the knowledge of patients with asthma regarding the pathology, before and after an educational intervention. This is a descriptive cross-sectional study with a qualitative approach through the application of questionnaires. The inclusion criteria were patients aged 18 to 60 years attended by an Outpatient Center with the diagnosis of asthma registered in the medical records and who agreed to participate in the study in all its phases. Those who did not consent to answer the questionnaire, and / or who had cognitive impairment and with pulmonary diagnoses other than asthma were excluded. The study showed that patients who attended the meetings obtained positive results, such as improving knowledge and managing the disease. The holding of meetings with a small number of participants contributed to a greater adaptation of the topics addressed to the needs of each case, although it restricted the debate and the exchange of knowledge between patients.
\end{abstract}

Keywords: $\quad$ Asthma; Self-care; Health education. 


\section{INTRODUÇÃO}

A asma é uma condição crônica bastante comum que afeta pessoas de qualquer idade e é considerada um problema mundial, acometendo cerca de 300 milhões de indivíduos, tendo uma prevalência global em torno de $20 \%$ para o Brasil, representando a terceira causa de hospitalizações em crianças, causada por exacerbação de seus sintomas (SBPT, 2012; CORIOLANO, et al., 2011; GINA, 2017). A asma tem alta morbidade, gerando elevados custos para o sistema de saúde e impactando negativamente a qualidade de vida dos doentes e de seus familiares (SBPT, 2012).

A asma é definida por uma doença inflamatória crônica associada à hiperresponsividade das vias aéreas e obstrução ao fluxo aéreo, e seu quadro clínico é caracterizado por episódios recorrentes de sibilos, dispneia, opressão torácica e tosse (CORIOLANO et al., 2011; SBPT, 2012).

No controle da asma as medidas educativas compõem, juntamente com o adequado tratamento farmacológico, os pilares de sustentação do tratamento visando diminuir exacerbações. 0 conhecimento do paciente e/ou de seus familiares a respeito da asma, abrangendo a fisiopatologia da doença, reconhecimento do quadro clínico, os desencadeadores das crises, o uso correto dos dispositivos inalatórios, conhecimento da complexidade dos esquemas terapêuticos e conhecimento das atitudes a tomar frente à exacerbação dos sintomas influenciam positivamente no tratamento dos asmáticos (OLIVEIRA et al., 2002; SBPT, 2012).

Segundo a IV Diretrizes Brasileiras para o Manejo da Asma, de 2006, a educação associada ao tratamento farmacológico constitui um dos pilares fundamentais no tratamento da asma. Percebe-se que a educação do paciente/família constitui uma medida facilitadora na prevenção de exacerbação de sintomas e melhora do quadro. Assim, reduzindo as visitas de emergência, diminuindo perda de dias de escola e de trabalho, reduzindo custos econômicos (DIRETRIZES, 2006).

Os grupos focais se revelam especialmente oportunos em estudos de análise de implantação de programas e ações de saúde nos quais se pretende valorizar a opinião e a percepção de sujeitos diretamente envolvidos com o objeto a ser avaliado. Ademais, os grupos focais demonstram ser espaços privilegiados de discussão e de trocas de experiências, com formato que estimula 0 debate entre os participantes, permitindo que os temas abordados sejam mais problematizados. Dessa forma, favorecem a reflexão crítica e coletiva e a identificação de consensos ou divergências significativas sobre os pontos abordados (TRAD, 2009).

A educação sobre asma tem se restringido às abordagens simplificadas, destinados a focar o conteúdo em aspectos essenciais, o que provavelmente tem limitado os resultados de saúde em adultos com asma, uma vez que apenas as informações a respeito do assunto não têm se mostrado suficiente (PLAZA et al., 2015).

Segundo o Global Initiative for Asthma (GINA), o controle da asma atualmente é deficiente o que prevê um futuro com fraco controle. No Brasil, apenas 9,3\% dos pacientes asmáticos estão controlados; $56,5 \%$ parcialmente controlados e, $34,2 \%$ controlados. Esse dado pode ser devido a existência de poucos centros de saúde e profissionais que oferecem programas de educação aos seus pacientes com asma, embasando a justificativa do presente trabalho (PLAZA et al., 2015).

Como hipótese, espera-se encontrar falta de conhecimento sobre asma entre os pacientes do Centro Ambulatorial Dr. Romes Nader, que incluem um desconhecimento sobre sintomas da doença, seus fatores desencadeantes e a identificação de exacerbações precocemente, bem como uma má adesão ao tratamento medicamentoso e não medicamentoso.

Tem-se como objetivo geral analisar o conhecimento dos pacientes portadores de asma brônquica a respeito da patologia e estabelecer uma intervenção educativa em asma em pacientes cadastrados em um centro ambulatorial da cidade de Araguari - MG.

Os objetivos específicos consistem em avaliar o conhecimento dos pacientes asmáticos sobre a doença, propiciar um ambiente para os pacientes compartilharem informações, por meio de discussões estruturada por grupo focal, estabelecer uma intervenção educativa para elevar o nível de conhecimento dos participantes e analisar os resultados da intervenção. 
2

REVISÃO DE LITERATURA

Asma é uma doença inflamatória crônica das vias aéreas, a qual está associada à hiperresponsividade das vias aéreas, que leva a episódios recorrentes de sibilos, dispneia, opressão torácica e tosse, particularmente à noite ou no início da manhã. Esses episódios são uma consequência da obstrução ao fluxo aéreo intrapulmonar generalizada e variável, reversível espontaneamente ou com tratamento (GINA, 2017).

É uma doença que afeta aproximadamente 358 milhões de indivíduos, tornando-se um grave problema de saúde com crescente prevalência em todo o mundo. A maioria dos afetados estão em países de baixa e média renda, presente em pessoas de todas as idades. Em 2015, mais de 397.100 mortes foram causadas por asma e a maior parte ocorreu nos países em desenvolvimento (AYELE; TEGEGN, 2017).

O custo direto da asma o qual engloba a utilização de serviços de saúde e medicações, é o dobro entre pacientes com asma não controlada comparado aos que estão com asma controlada, sendo a falta de controle da asma o maior componente relacionado à utilização dos serviços de saúde. Além disso, o custo indireto (número de dias perdidos de escola e trabalho, por exemplo) é superior no grupo com asma não controlada, sendo que o custo da asma aumenta proporcionalmente com a gravidade da doença (SBPT, 2012).

Os gastos com asma grave consomem quase $25 \%$ da renda familiar dos pacientes da classe menos favorecida, sendo que a recomendação da Organização Mundial de Saúde é de que esse montante não exceda a $5 \%$ da renda familiar (SBPT, 2012).

Os sintomas da asma são sibilos, dispneia, opressão torácica e tosse, sobretudo nas primeiras horas da manhã, no entanto, variam ao longo do tempo quanto à intensidade, ocorrência e frequência. Os sintomas estão associados a dificuldade para exalar o ar dos pulmões devido à broncoconstrição, espessamento da parede das vias aéreas e aumento da produção de muco. Podem ser desencadeados ou agravados por fatores como infecções virais, exposição à alérgenos, à fumaça e cigarro, exercício e estresse (GINA, 2017).

O diagnóstico da asma baseia-se em três pilares: os dados clínicos obtidos pela anamnese, a identificação da sensibilidade alérgica e, em crianças maiores e adultos, em parâmetros de função pulmonar (SILVA, 2008).

O diagnóstico clínico da asma é sugerido quando o paciente apresenta um ou mais sintomas da doença. Embora esse diagnóstico, considerando a forma clássica de apresentação, não seja difícil, a confirmação deve ser feita por um método objetivo, uma vez que os sinais e sintomas da asma não são exclusivos dessa condição. Os testes diagnósticos disponíveis na prática clínica incluem espirometria (antes e após o uso de broncodilatador), testes de broncoprovocação, e medidas seriadas do pico de fluxo expiratório (PFE). Em certos casos, a comprovação da reversibilidade da obstrução ao fluxo aéreo pode ser demonstrada apenas com o teste terapêutico com corticoide oral (GINA, 2017).

A espirometria é o exame complementar de maior importância na avaliação da asma, apresentando três utilidades principais: estabelecer o diagnóstico, documentar a gravidade da obstrução ao fluxo aéreo e monitorar o curso da doença e as modificações decorrentes do tratamento. Entretanto, a espirometria normal não exclui o diagnóstico; nesses casos, a observação da variabilidade do pico de fluxo expiratório (PFE), a repetição da espirometria durante um período sintomático ou um teste de broncoprovocação pode confirmar ou afastar a suspeita de asma (TABALIPA; SILVA, 2012).

Os principais diagnósticos diferenciais em crianças acima de cinco anos e adultos são: rinossinusite, síndrome de hiperventilação alveolar, síndrome do pânico, obstrução de vias aéreas superiores (neoplasias e aspiração de corpos estranhos), disfunção das cordas vocais, DPOC e outras doenças obstrutivas das vias aéreas inferiores (bronquiolites, bronquiectasias e fibrose cística), doenças difusas do parênquima pulmonar, doenças da circulação pulmonar (hipertensão e embolia) e insuficiência cardíaca sistólica e diastólica (SBPT, 2012).

De acordo com as IV Diretrizes para o Manejo da Asma, os objetivos principais do tratamento da asma são controlar os sintomas, prevenir a limitação crônica ao fluxo aéreo, secundária ao remodelamento brônquico, permitir atividades normais (trabalho, escola e lazer), manter a função pulmonar normal ou a melhor possível, evitar crises, idas à emergência e hospitalizações, reduzir a necessidade do uso de broncodilatador para alívio, 
minimizar efeitos adversos da medicação e prevenir a morte (DIRETRIZES, 2006).

O tratamento de manutenção baseia-se em medidas de controle ambiental, para diminuir a exposição aos alérgenos e irritantes inaláveis, farmacoterapia, para redução da inflamação e hiperresponsividade brônquica e, em casos selecionados, imunoterapia, para reduzir a sensibilidade alérgica. Além disso, o tratamento de doenças e condições associadas, como rinossinusite, Doença do Refluxo Gastroesofágico (DRGE), obesidade, ansiedade e depressão ajudam o controle da doença (SILVA, 2008).

Os medicamentos para asma se dividem em:

a) medicamentos de manutenção, usados para prevenir as crises e controlar a doença: corticosteroides inalatórios ( $\mathrm{Cl})$, e sistêmicos, cromonas (cromoglicato dissódico), antagonistas de receptores de leucotrienos, beta2-agonistas de longa duração (formoterol e salmeterol), usados em conjunto com os $\mathrm{Cl}$, teofilina de liberação lenta, bambuterol e, mais recentemente, o anticorpo anti-lgE.

b) medicamentos para alívio dos sintomas agudos: beta2-agonistas com rápido início de ação (salbutamol, fenoterol), brometo de ipratrópio, aminofilina, corticosteroides sistêmicos (hidrocortisona, prednisolona) (DIRETRIZES, 2006).

O tratamento deve ser iniciado de acordo com a classificação da gravidade da doença. Uma vez obtido o controle por período de três meses (nos casos leves) a seis meses (nos casos moderados a graves), pode-se tentar reduzir as doses das medicações, mantendo-se o paciente monitorizado e sempre deixando os corticoides inalatórios por último (SILVA, 2008).

\section{3}

\section{METODOLOGIA}

Trata-se de um estudo descritivo de corte transversal, com abordagem quanti-qualitativa, realizado no período de fevereiro a maio de 2019 por meio da estruturação de grupo focal (GF) e aplicação de questionários elaborados pelos autores deste trabalho. O trabalho foi composto pelas seguintes etapas: aplicação de um questionário sociodemográfico e outro relacionado aos conhecimentos gerais sobre asma antes da primeira reunião do GF, em caráter privativo. Posteriormente à segunda reunião, foi realizada uma medida educativa por meio de uma exposição dialogada sobre temas pertinentes da exacerbação e controle da asma. Logo após a aula educativa foi aplicado novamente o questionário sobre asma. As etapas supracitadas ocorreram nas dependências do Centro Ambulatorial Dr. Romes Nader no município de Araguari-MG.

O questionário sociodemográfico foi composto por questões relacionadas ao gênero, faixa etária, escolaridade, situação ocupacional, renda mensal, aspectos relacionados à residência, composição familiar e à saúde do paciente. Já o questionário sobre conhecimentos gerais sobre asma, envolveu a definição do quadro de asma, fatores de exacerbação e uso correto da medicação.

Como critérios de inclusão do estudo, foram selecionados voluntários entre 18 a 60 anos, que estavam em atendimento pelo Centro Ambulatorial no momento da investigação, com diagnóstico de asma registrado em prontuário e que concordaram em participar do estudo em todas as suas fases. Foram excluídos aqueles pacientes que não consentirem em participar da pesquisa, os que apresentarem déficit cognitivo, e/ou com outros diagnósticos pulmonares que não asma. Obteve-se como amostra final do estudo 5 voluntários.

Em seguida, os dados foram coletados por técnica específica de GF, caracterizado como um grupo que se reúne para problematizar a respeito de um tema específico em comum aos participantes. A discussão foi gravada por meio de áudio, e suas partes mais importantes foram utilizadas na construção da aula educativa e do trabalho. A escolha dessa técnica de coleta de dados decorreu de sua possibilidade de promover a interação grupal horizontalizada entre os participantes, que permite explorar e ampliar a compreensão em torno do fenômeno investigado e, principalmente, a troca de experiências entre integrantes acerca do tema tratado.

O GF foi composto por 1 moderador, 2 observadores e 5 participantes. Em relação ao conteúdo tratado foi elaborado um roteiro de entrevista, aplicado pelo moderador contendo questões do tipo: introdutória, de transição, chave, final, resumo e de fechamento. No entanto, os participantes puderam tratar de diferentes assuntos, abarcando conceitos e preconceitos, opiniões e ideias, valores, sentimentos e ações, voltados para o objetivo da pesquisa. 
Para a compilação dos dados, os autores do trabalho elegeram pseudônimos aos membros do GF para preservar suas identidades. Por motivo de segurança, no dia anterior a cada evento, foi realizado contato com os participantes para confirmar sua presença. Foram realizados 3 encontros com os participantes, sendo 2 reuniões do GF com duração de 90 minutos, e uma 1 expositiva após a segunda reunião do GF.

A medida educativa foi realizada por meio de aula/palestra em um encontro com os pacientes, abordando os temas: controle dos fatores ambientais desencadeadores da asma e medidas para prevenir crises da doença, bem como uso correto de medicamentos, e como reconhecer e agir durante uma crise de asma.

Como forma de evitar possíveis vieses no recrutamento dos pacientes, foi observado aqueles que não pertencem à um mesmo círculo de amizade, e aqueles que possuem algum grau de parentesco. Para os usuários asmáticos houve a necessidade de os prontuários estarem com informações fidedignas quanto a doença. Todas as informações coletadas foram registradas no roteiro, sendo preservada a identidade de cada usuário. Durante a análise dos dados, a abordagem metodológica utilizada foi de um levantamento descritivo para organização dos dados coletados.

Os benefícios deste trabalho consistiram nos pacientes asmáticos vivenciarem um momento de aprofundar seus conhecimentos sobre a asma, principalmente a troca de experiências acerca do cuidado e controle com a doença, reconhecimento de episódios de exacerbações e seus fatores desencadeantes.

O risco consistiu no portador se sentir constrangido quando questionado sobre seus conhecimentos em asma. Ademais, foi previsto pelos pesquisadores a possibilidade de intercorrências clínicas, como por exemplo crise asmática, dos pacientes no momento da investigação. Caso isso ocorresse, o Serviço de Atendimento Móvel de Urgência (SAMU) e/ou o serviço de emergência do Corpo de Bombeiros Militares do munícipio de Araguari seriam acionados através do telefone 192 e/ou 193, respectivamente, determinando a paralisação do GF, com posterior agendamento dele. No presente estudo nenhum dos investigados na pesquisa tiveram intercorrências durante a condução do GF.
Os participantes foram encaminhados para responder os questionários após serem devidamente identificados como integrantes do projeto e não tiveram seus nomes escritos nos instrumentos de avaliação, a fim de não gerar constrangimentos. Os dados quantitativos relacionados ao perfil sociodemográfico dos entrevistados e os conhecimentos sobre mitos relacionados à asma tiveram uma abordagem descritiva.

A pesquisa foi realizada de acordo com os princípios éticos, com o consentimento dos envolvidos para participação no GF, coleta das informações e aplicação dos questionários. Ademais, a participação no GF e preenchimento dos questionários só foram realizados após compactuação do sujeito da amostra por meio do preenchimento do Termo de Consentimento Livre e Esclarecido (TCLE). Salienta-se que o estudo foi realizado somente após liberação do protocolo de aprovação do Comitê de Ética em Pesquisa (CEP), sob protocolo $n=3.157 .752$.

\section{RESULTADOS E DISCUSSÃO}

A população estimada do estudo foi selecionada após consulta prévia aos prontuários do Centro Ambulatorial até junho de 2018, totalizando 69 pacientes que atenderam aos critérios de inclusão do estudo.

Para constituição da amostra não probabilística de conveniência, foram selecionados dentre estes um total de 15 pacientes definidos por meio de sorteio aleatório, satisfazendo as técnicas e procedimentos exigidos pelo GF.

Mediante a desatualização do cadastro registrado no banco de dados do Centro Ambulatorial, não foi possível entrar em contato com alguns pacientes listados. Associado a isso, diante da baixa adesão dos voluntários de asma, apenas 12 demonstraram interesse em participar do estudo, e destes, apenas 5 compareceram no primeiro encontro, já no segundo encontro e na aula educativa apenas 2 compareceram. 
Fluxograma 1 - Delineamento do percurso metodológico de seleção da amostra do estudo.

Amostra inicial: 69 pacientes do Ambulatório Doutor Romes Nader

Sorteio de 15 pacientes para grupo focal

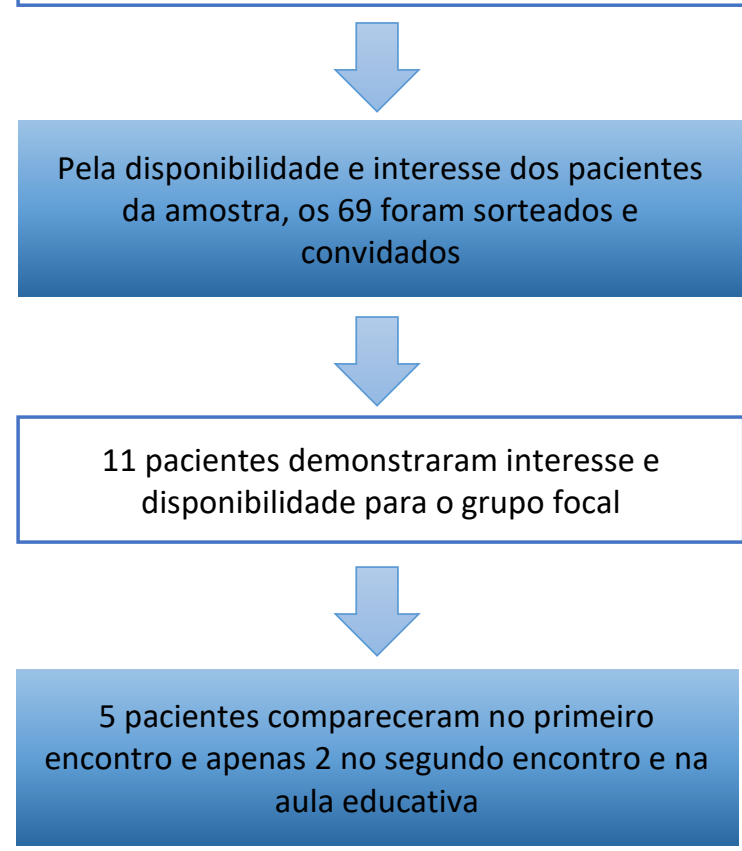

Fonte: os autores

A ocorrência de baixa adesão a esquemas terapêuticos em doenças crônicas está bem documentada, com cerca de apenas $50 \%$ dos pacientes agindo estritamente conforme as orientações recebidas da equipe de profissionais da saúde, seja em regimes preventivos ou terapêuticos (CHATKIN, 2006). Isso foi observado durante o estudo, com demonstração clara da baixa adesão ao tratamento e do ínfimo interesse em políticas educativas. Desse modo, o presente estudo apresenta limitações quanto a validade externa, devido ao tamanho da amostra.

Foram realizados duas (2) sessões de grupo focal, com duração de aproximadamente 90 minutos cada, e uma aula educativa ministrada pelos pesquisadores, com aplicação do questionário antes do início da primeira reunião e após a aula educativa. Ao longo da primeira sessão, foram discutidos a visão individual de cada paciente sobre o conceito de asma, percepção de crise, fatores desencadeantes e as principais dúvidas relacionadas à doença. Um ponto de destaque foi o interesse da maioria dos participantes sobre prática de exercícios respiratórios por meio da Yoga para alívio dos sintomas respiratórios. Já na segunda sessão, o foco se voltou para o tratamento da doença, com discussões sobre os dispositivos usados, o modo de utilização e o medicamento para controle e resgate. Ficou nítido como é importante a individualização do tratamento desses pacientes, pois cada um apresentava uma frequência e graus de intensidade distintos de sintomas, com consequente variação dos métodos de tratamento, sendo a adesão ao tratamento um dos itens fundamentais para a melhoria de qualidade de vida.

No decorrer dos GF's houve uma referência constante à dispneia frequente e os sintomas desencadeados por poeira e cheiros fortes. Outro ponto relevante, foi a realização ou não de atividade física para melhora do quadro respiratório, com divisão das opiniões dos participantes. Após o término das sessões do GF, foi realizada uma aula expositiva abordando as principais dúvidas percebidas ao longo das reuniões e as formas corretas de realizar os tratamentos não farmacológicos e farmacológicos. Após a aula, os questionários foram reaplicados.

Nesse contexto, houve reforço dos principais fatores desencadeantes das crises asmáticas e da importância do controle ambiental e da limpeza correta da casa para garantir um bom controle dessa doença crônica. Além disso, foi apresentado a relevância da atividade aeróbica supervisionada e vídeos demonstrando o uso correto da medicação de controle e resgate, além da forma de manusear e utilizar o dispositivo. Os estudos sobre o impacto a longo prazo da Yoga nos pacientes que possuem asma são escassos e pouco pode ser afirmado sobre o tema, porém tem mostrado relevância semelhante ao exercício aeróbico no controle sintomático.

No que tange os questionários aplicados durante o estudo, utilizou-se um questionário demográfico e socioeconômico e um para avaliação do conhecimento do paciente em relação à asma. Os dados demográficos e socioeconômicos se encontram na tabela 1 , juntamente com os dados sobre a saúde (tabelas 2 e 3 ) dos pacientes colhidos no mesmo questionário. 
Tabela 1 - Características sociodemográficas dos participantes dos grupos focais no Ambulatório Dr. Romes Nader, em Araguari - MG.

\begin{tabular}{lc}
\hline Variáveis & Frequência \\
\hline Gênero & \\
Feminino & $60 \%$ \\
Masculino & $40 \%$ \\
\hline Faixa etária & \\
\hline $35-39$ anos & $20 \%$ \\
\hline $40-44$ anos & $40 \%$ \\
\hline $45-50$ anos & $40 \%$ \\
Outras faixas etárias & $0 \%$ \\
\hline Escolaridade & \\
\hline E. Fundamental Incompleto & $20 \%$ \\
\hline E. Fundamental Completo & $40 \%$ \\
\hline E. Médio Incompleto & $20 \%$ \\
\hline E. Médio Completo & $20 \%$ \\
\hline Outros níveis de escolaridade & $0 \%$ \\
\hline Ocupação & \\
\hline Ativo & $80 \%$ \\
\hline Inativo & $20 \%$ \\
\hline Renda Mensal & \\
\hline 1-3 salários & $80 \%$ \\
\hline Acima de 3 salários & $20 \%$ \\
\hline Outras rendas & $0 \%$ \\
\hline
\end{tabular}

Fonte: os autores

Tabela 1 - Principais doenças crônicas, eventos cardiovasculares, quedas e hospitalizações dos participantes dos grupos focais no Ambulatório Dr. Romes Nader, em Araguari - MG.

\begin{tabular}{lc}
\hline \multicolumn{1}{c}{ Quadro clínico } & $\begin{array}{c}\text { Pacientes que o } \\
\text { apresentam ou } \\
\text { apresentaram }\end{array}$ \\
\hline Diabetes & $0 \%$ \\
\hline AVE & $0 \%$ \\
\hline HAS & $0 \%$ \\
\hline Insônia & $40 \%$ \\
\hline Reumatismo & $0 \%$ \\
\hline Varizes & $20 \%$ \\
\hline Quedas & $40 \%$ \\
Hospitalizações & $40 \%$ \\
\hline IAM & $0 \%$ \\
\hline Saúde mental na família & $40 \%$ \\
\hline
\end{tabular}

Fonte: os autores

Segundo informações divulgadas pelo portal do Ministério da Saúde (2017), a educação do paciente é parte fundamental da terapêutica da asma e deve integrar todas as fases do atendimento ambulatorial e hospitalar.
Deve-se levar em conta aspectos culturais e abranger aspectos de compreensão a respeito da doença. Assim, nos questionários aplicados sobre avaliação do conhecimento, buscou-se informações sobre o grau de esclarecimento dos pacientes sobre o conceito da doença, o uso correto das medicações, os fatores desencadeantes e de piora, o reconhecimento da crise e as atitudes a serem tomadas quando ela ocorre. Foram aplicados antes do início do primeiro encontro e após a intervenção educativa.

Tabela 2 - Percepção acerca da visão, audição e saúde, e comparação entre sua saúde e de seus pares dos participantes dos grupos focais no Ambulatório Dr. Romes Nader, em Araguari - MG.

\begin{tabular}{lccc}
\hline \multicolumn{1}{c}{ Percepção } & Ótima & Ruim & Regular \\
\hline Visão & $20 \%$ & $40 \%$ & $40 \%$ \\
Audição & $80 \%$ & $0 \%$ & $20 \%$ \\
$\begin{array}{l}\text { Saúde } \\
\text { Comparação } \\
\text { entre sua saúde } \\
\text { e de seus pares }\end{array}$ & $\begin{array}{c}\text { Muito } \\
\text { melhor }\end{array}$ & $\begin{array}{c}\text { Mais ou } \\
\text { menos }\end{array}$ & $\begin{array}{c}\text { Muito } \\
\text { pior }\end{array}$ \\
& $40 \%$ & $40 \%$ & $20 \%$ \\
\hline \multicolumn{4}{c}{ Fonte: os autores } \\
\hline
\end{tabular}

No primeiro encontro, os 5 pacientes presentes responderam aos questionários. Questionou-se sobre o conceito de asma, o conceito de broncoespasmo, os conhecimentos gerais sobre asma e os passos de uso do dispositivo inalatório. Questionou-se também a respeito dos cuidados domésticos, dentre eles os fatores que pioram a asma, a lavagem das mãos quando há alguém gripado no domicílio, a limpeza dos móveis, o uso do fogão adequado, que seria o fogão a gás em detrimento do fogão a lenha, a existência de agentes de saúde para pacientes com asma.

Perguntou-se também sobre os fatores que podem desencadear uma crise de asma, os cuidados com animais domésticos, cuidados com a limpeza da casa, sinais e sintomas da crise, ações a serem realizadas ao notar a crise, uso de medicações, função dos broncodilatadores e dos corticoides, efeitos colaterais dos corticoides e as medicações que devem ser usadas na crise (tabelas 4 e 5). 
Tabela 3 - Conhecimento dos principais termos que definem a asma, uso do principal dispositivo para controle da doença e fatores de risco para desencadeamento de exacerbações de crises dos participantes dos grupos focais no Ambulatório Dr. Romes Nader, em Araguari MG.

\begin{tabular}{lc}
\hline \multicolumn{1}{c}{ Questões } & Acertos \\
\hline Conceito de asma & $60 \%$ \\
\hline $\begin{array}{l}\text { Conceito de broncoespasmo } \\
\text { Conhecimentos gerais sobre asma }\end{array}$ & $30 \%$ \\
\hline Passo-a-passo uso do dispositivo inalatório & 1 a 3 \\
\hline Fatores que pioram a asma & erros \\
\hline $\begin{array}{l}\text { Lavagem das mãos quando alguém está } \\
\text { gripado no domicílio }\end{array}$ & $83 \%$ \\
\hline $\begin{array}{l}\text { Limpeza dos móveis } \\
\text { Uso do fogão adequado }\end{array}$ & $80 \%$ \\
\hline $\begin{array}{l}\text { Existência de atividades de agentes de } \\
\text { saúde voltadas para asma }\end{array}$ & $86 \%$ \\
\hline $\begin{array}{l}\text { Fatores que desencadeiam crise } \\
\text { Cuidados com animais domésticos }\end{array}$ & $93 \%$ \\
\hline $\begin{array}{l}\text { limpeza da casa } \\
\text { Reconhecimento de sinais e sintomas da } \\
\text { crise }\end{array}$ & $83 \%$ \\
\hline $\begin{array}{l}\text { Ações diante da crise } \\
\text { Função dos broncodilatadores }\end{array}$ & $90 \%$ \\
\hline $\begin{array}{l}\text { Função dos corticoides } \\
\text { Efeitos colaterais dos corticoides }\end{array}$ & $73 \%$ \\
\hline \begin{tabular}{l} 
Medicações que devem ser utilizadas na \\
\hline
\end{tabular} & $70 \%$ \\
\hline
\end{tabular}

Fonte: os autores

Os medicamentos utilizados pelos pacientes são de forma geral corticoides inalatórios e broncodilatadores e cada paciente faz uso de um tipo diferente de dispositivo. Após a intervenção educativa, reaplicou-se o questionário de conhecimento dos pacientes. Em decorrência do baixo comparecimento no segundo encontro e na intervenção não se pode inferir uma real melhora do conhecimento dos pacientes, mesmo que em porcentagens tenha havido mais acertos em algumas questões.

Nota-se que, tanto no primeiro encontro quanto após a intervenção, que os pacientes ainda não têm conhecimento satisfatório principalmente sobre o conceito da doença e sobre as medicações utilizadas. Além disso, percebe-se que existem muitas questões que ainda suscitam dúvidas, como por exemplo sobre lavagem das mãos, o uso de alguns produtos de limpeza e alguns pontos de conhecimento geral sobre a asma.

Tabela 4 - Conhecimento dos principais termos que definem a asma, uso do principal dispositivo para controle da doença e fatores de risco para desencadeamento de exacerbações de crises dos participantes dos grupos focais no Ambulatório Dr. Romes Nader, em Araguari MG.

\begin{tabular}{lc}
\hline \multicolumn{1}{c}{ Questões } & Acertos \\
\hline Conceito de asma & $62 \%$ \\
\hline Conceito de broncoespasmo & $50 \%$ \\
\hline $\begin{array}{l}\text { Conhecimentos gerais sobre asma } \\
\text { Passo-a-passo uso do dispositivo inalatório }\end{array}$ & $\begin{array}{c}1 \text { a } 3 \\
\text { erros }\end{array}$ \\
\hline Fatores que pioram a asma & $100 \%$ \\
\hline $\begin{array}{l}\text { Lavagem das mãos quando alguém está } \\
\text { gripado no domicílio }\end{array}$ & $75 \%$ \\
\hline $\begin{array}{l}\text { Limpeza dos móveis } \\
\text { Uso do fogão adequado }\end{array}$ & $100 \%$ \\
\hline $\begin{array}{l}\text { Existência de atividades de agentes de } \\
\text { saúde voltadas para asma }\end{array}$ & $100 \%$ \\
\hline $\begin{array}{l}\text { Fatores que desencadeiam crise } \\
\text { Cuidados com animais domésticos }\end{array}$ & $100 \%$ \\
\hline limpeza da casa & $83 \%$ \\
\hline $\begin{array}{l}\text { Reconhecimento de sinais e sintomas da } \\
\text { crise }\end{array}$ & $95 \%$ \\
\hline $\begin{array}{l}\text { Ações diante da crise } \\
\text { Função dos broncodilatadores }\end{array}$ & $100 \%$ \\
\hline $\begin{array}{l}\text { Função dos corticoides } \\
\text { Efeitos colaterais dos corticoides }\end{array}$ & $75 \%$ \\
\hline $\begin{array}{l}\text { Medicã̃es que devem ser utilizadas na } \\
\text { crise }\end{array}$ & $66 \%$ \\
\hline
\end{tabular}

Fonte: os autores

Em decorrência da pequena amostra, fazer correlações entre os dados demográficos e socioeconômicos com os dados a respeito do conhecimento dos pacientes de forma estatística não gera grandes inferências. $40 \%$ dos pacientes tem ensino médio completo o que pode influenciar no nível de instrução dos voluntários sugerindo a forma de como direcionar o GF e a aula educativa, e $80 \%$ tem renda entre 1 e 3 salários-mínimos e a maioria refere ter boa saúde.

Após a intervenção educativa, $100 \%$ dos entrevistados demostraram saber reconhecer a crise após, e $75 \%$ destes possuíam conhecimento efetivo de como agir diante dela e $62 \%$ sabiam qual 
medicação utilizar nestes episódios. Ao se observar as medidas usadas para tratamento medicamentoso, $66 \%$ dos pacientes conheciam a função de um broncodilatador, $50 \%$ conheciam a função dos corticoides, sendo que deste último não mais que $50 \%$ conheciam seus efeitos colaterais. Desta forma, evidencia-se a deficiência do saber principalmente com relação ao tratamento da asma, o que dificulta ainda mais adesão ao mesmo, visto que os pacientes demonstram não ter grande consciência da função de cada medicamento e da forma de utilizá-los.

\section{CONCLUSÕES}

O presente estudo demonstra que os pacientes que participaram das reuniões obtiveram resultados positivos, como a melhoria do conhecimento e da gestão da doença.

A realização das reuniões com um pequeno número de participantes contribuiu para maior adaptação dos temas abordados às necessidades de cada caso, embora em contrapartida tenha restringido o debate e a troca de conhecimento entre os pacientes. A falta de adesão dos pacientes as reuniões, deve ser considerada como principal fator limitador para o presente estudo. Tal limitação impede que os resultados obtidos sejam utilizados para a análise de uma maior população de pacientes asmáticos.

Pode-se inferir que este estudo possui limitações, quando se trata de sua proposta inicial para avaliação do conhecimento dos pacientes portadores de asma para com sua patologia, já que houve uma baixa adesão dos asmáticos quando convidados a participar das reuniões do grupo focal. No entanto, através desta pesquisa podemos reafirmar como é insuficiente a busca por esclarecimentos a respeito da própria patologia quando se trata dos acometidos, já que de todos os participantes previamente selecionados, um número muito inferior compareceu aos encontros, e dentro deste grupo, dúvidas básicas sobre o manejo da doença foram observadas.

Será importante organizar estudos que explorem os fatores que influenciam a aceitação dos programas de educação em saúde, para que sejam criadas estratégias por meio das equipes da atenção primária que permitem ultrapassar esta dificuldade de adesão inicial aos programas.
A principal implicação prática do presente estudo consiste em incentivar a adoção de medidas educativas no cotidiano médico, compondo desde as consultas da atenção primária às de nível avançado, visto que a maioria dos pacientes não possuem controle de suas patologias por não conhecerem sua doença. A falta de conhecimento dos conceitos básicos, dos fatores desencadeantes de uma crise, bem como preveni-la e agir diante da mesma lançando mão tanto do tratamento medicamento como o não medicamentoso leva ao não controle da doença.

\section{REFERÊNCIAS}

AYELE, A. A.; TEGEGN, H. G. Non adherence to inhalational medications and associated factors among patients with asthma in a referral hospital in Ethiopia, using validated tool TAI. Asthma Research and Practice, v.3, n.7, 2017. p. 3-7

BRASIL. Ministério da Saúde. Asma: o que é, causas, sintomas, tratamento, diagnóstico e prevenção.

2017. Disponível em:

$<$ http://www.saude.gov.br/saude-de-az/asma\#tinicio>. Acesso em: 14 jun 2019.

CHATKIN, José Miguel et al. Adesão ao tratamento de manutenção em asma (estudo ADERE). J Bras Pneumol, v. 32, n. 4, p. 277-83, 2006.

CORIOLANO, M. W. L. et al. Repercussão de uma intervenção educativa com agentes comunitários de saúde nas condições ambientais de domicílios de crianças asmáticas. J bras pneumol, v. 37, n. 3, 2011. p. $317-25$

GINA. Global Strategy for Asthma Management and Prevention. Global Initiative for Asthma (GINA), 2017. Disponível em: < http://ginasthma.org/download/317/>. Acesso: 09 de outubro de 2017.

IV DIRETRIZES BRASILEIRAS PARA O MANEJO DA ASMA. J. bras. pneumol. São Paulo, v. 32, supl. 7, p. S447-S474, nov. 2006.

OLIVEIRA, M. A. et al. Custo-efetividade de programa de educação para adultos asmáticos atendidos em hospital-escola de instituição pública. J.

Pneumologia, São Paulo, v. 28, n. 2, 2002. p. 71-76. 
PLAZA, V. et al. A repeated short educational intervention improves asthma control and quality of life. European Respiratory Journal. v.46, n. 5, 2015. p. 1298-1307.

SILVA, E. C. F. Asma Brônquica. Revista do Hospital Universitário Pedro Ernesto - UERJ, Rio de Janeiro, ano 7, jul./dez. 2008.

\section{SOCIEDADE BRASILEIRA DE PNEUMOLOGIA E}

TISIOLOGIA - SBPT. Diretrizes da Sociedade Brasileira de Pneumologia e Tisiologia para o Manejo da Asma - 2012. J. Bras pneumol, v. 38, suplemento 1, p.S1S46, 2012.

TABALIPA, F. O.; SILVA, J. Como diagnosticar e tratar asma. Revista Brasileira de Medicina, v. 69, n. 12, 2012, p. 83-96.

TRAD, L. A. B. Grupos focais: conceitos, procedimentos e reflexões baseadas em experiências com o uso da técnica em pesquisas de saúde. Physis: revista de saúde coletiva, v. 19, n.3, 2009, p. 777-796. 